Supplementary information for:

Real-time synchrotron PXRD study of the anti-site defect formation during sub- and supercritical synthesis of $\mathrm{LiFePO}_{4}$ and $\mathrm{LiFe}_{1-x \mathrm{Mn}_{\times} \mathrm{PO}_{4} \text { nanoparticles }}$

Kirsten Marie Ørnsbjerg Jensen, Mogens Christensen, Christoffer Tyrsted and Bo Brummerstedt Iversen

Center for Materials Crystallography, Department of Chemistry and iNano, Aarhus University,DK-8000 Århus C, Denmark

E-mail: $\underline{b o @ c h e m . a u . d k}$ 
Rietveld refinementsFigure S1. Representative Rietveld fit to the data. The observed intensities are plotted together with the intensities calculated from the model. The lower line shows the difference between the two. The frame was obtained during the experiment at $\mathrm{T}=170^{\circ} \mathrm{C}$, after 5 minutes of reaction time.

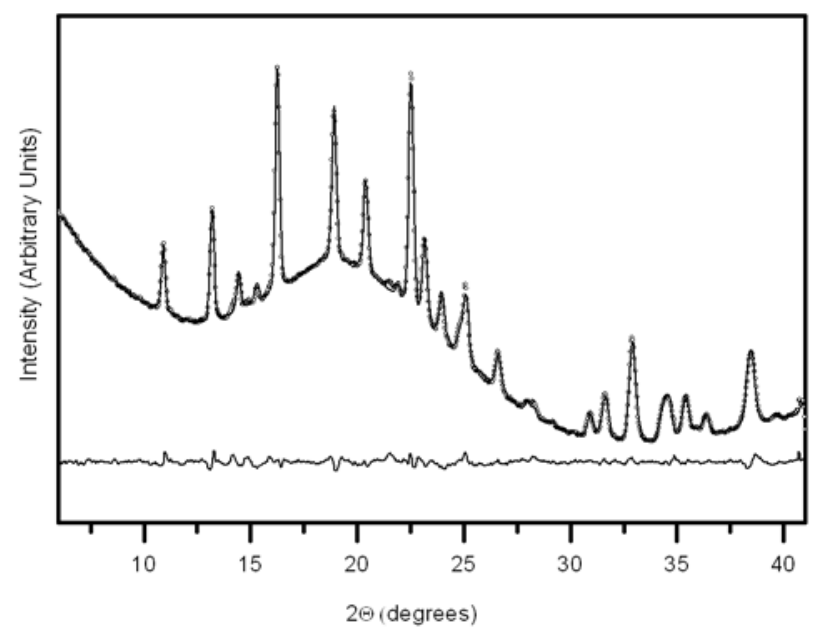

\begin{tabular}{|c|c|}
\hline Number of Bragg peaks & 64 \\
Number of data points & 1098 \\
\hline Number of background points & 24 \\
Total number of refined & 31 \\
parameters & \\
& \\
$\mathrm{R}_{\text {Bragg }}$ & $4.38 \%$ \\
$\mathrm{a}$ & $10.4524(2) \AA$ \\
$\mathrm{b}$ & $6.0512(2) \AA$ \\
$\mathrm{C}$ & $4.7524(2) \AA$ \\
Fe on Li site & $5.75(29) \%$ \\
$\mathrm{U}$ & $0.90(2)$ \\
$\mathrm{B}_{\text {overall }}$ & $1.46(9) \AA^{2}$ \\
\hline
\end{tabular}

Figure S2. Representative Rietveld fit to the data. The observed intensities are plotted together with the intensities calculated from the model. The lower line shows the difference between the two. The frame was obtained during the experiment at $\mathrm{T}=170^{\circ} \mathrm{C}$, after 15 minutes of reaction time.

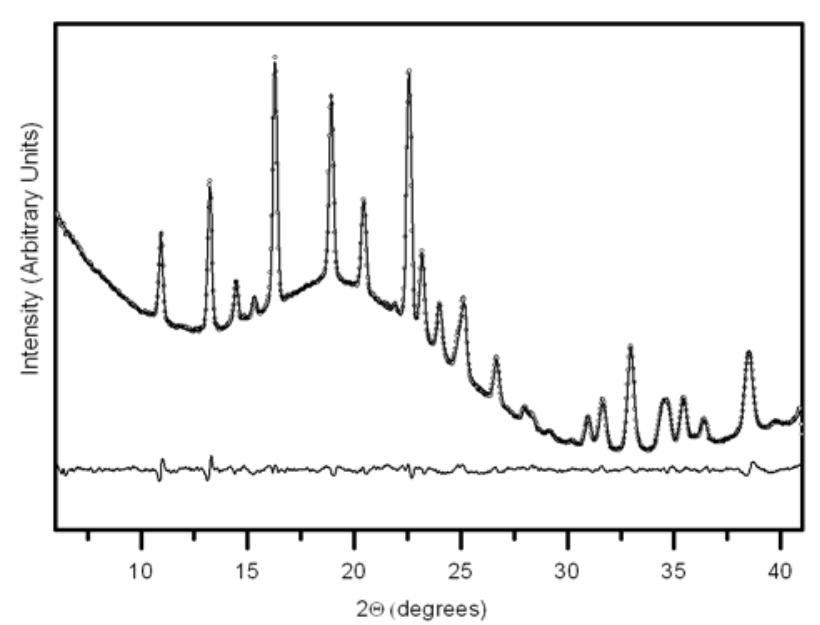

\begin{tabular}{|c|c|}
\hline Number of Bragg peaks & 64 \\
Number of data points & 1098 \\
\hline Number of background points & 24 \\
Total number of refined & 31 \\
parameters & \\
& \\
$\mathrm{R}_{\text {Bragg }}$ & $2.74 \%$ \\
$\mathrm{a}$ & $10.4312(1) \AA$ \\
$\mathrm{b}$ & $6.0513(2) \AA$ \\
$\mathrm{C}$ & $4.7405(1) \AA$ \\
Fe on Li site & $2.38(24) \%$ \\
$\mathrm{U}$ & $0.87(1)$ \\
$\mathrm{B}_{\text {overall }}$ & $1.46(6) \AA^{2}$ \\
\hline
\end{tabular}


Parameters found from refinement of the last frame in each series:

The atomic positions found by refinement of the last frame were used troughout the sequential refinement.

Last frame, $\mathrm{LiFePO}_{4}$, precursor $\mathrm{A}, \mathbf{1 7 0}^{\circ} \mathrm{C}$

\begin{tabular}{|l|l|}
\hline $\mathrm{R}_{\text {Bragg }}$ & $2.95 \%$ \\
\hline Number of data points & 1098 \\
\hline Number of background points & 24 \\
\hline Total number of refined parameters & 42 \\
\hline $\mathrm{a} / \AA$ & $10.4224(3)$ \\
\hline $\mathrm{b} / \AA$ & $6.0509(2)$ \\
\hline $\mathrm{c} / \AA$ & $4.7359(1)$ \\
\hline $\mathrm{B}_{\text {overall }} / \AA^{2}$ & $1.46(8)$ \\
\hline $\mathrm{U}$ (peak shape) & $0.82(1)$ \\
\hline
\end{tabular}

Atomic positions:

\begin{tabular}{|c|c|c|c|c|}
\hline Species & $\mathrm{x}$-coordinate & y-coordinate & z-coordinate & Occupancy \\
\hline Li1 & 0.00000 & 0.00000 & 0.00000 & $0.984(2)$ \\
\hline Fe1 & $0.28164(19)$ & 0.25000 & $0.9758(8)$ & $0.984(2)$ \\
\hline P1 & $0.0992(5)$ & 0.25000 & $0.4190(14$ & 1 \\
\hline $\mathrm{O} 1$ & $0.0932(14)$ & 0.25000 & $0.737(2)$ & 1 \\
\hline $\mathrm{O} 2$ & $0.4518(16)$ & 0.25000 & $0.2075(15)$ & 1 \\
\hline O3 & $0.1625(10)$ & $0.0440(15)$ & $0.2780(12)$ & 1 \\
\hline $\mathrm{Fe} 2$ & 0.00000 & 0.00000 & 0.00000 & $0.016(2)$ \\
\hline $\mathrm{Li} 2$ & $0.28164(19)$ & 0.25000 & $0.9758(8)$ & $0.016(2)$ \\
\hline
\end{tabular}

Last frame, $\mathrm{LiFePO}_{4}$, precursor $\mathrm{A}, 270^{\circ} \mathrm{C}$

\begin{tabular}{|l|l|}
\hline $\mathrm{R}_{\text {Bragg }}$ & $5.90 \%$ \\
\hline Number of data points & 1114 \\
\hline Number of background points & 24 \\
\hline Total number of refined parameters & 42 \\
\hline $\mathrm{a} / \AA$ & $10.3526(3)$ \\
\hline $\mathrm{b} / \AA$ & $6.0238(2)$ \\
\hline $\mathrm{c} / \AA$ & $4.7165(2)$ \\
\hline $\mathrm{B}_{\text {overall }} / \AA^{2}$ & $1.21(9)$ \\
\hline $\mathrm{U}$ (peak shape) & $0.74(1)$ \\
\hline
\end{tabular}

Atomic positions:

\begin{tabular}{|l|l|l|l|l|}
\hline Species & x-coordinate & y-coordinate & z-coordinate & Occupancy \\
\hline Li1 & 0.00000 & 0.00000 & 0.00000 & $0.990(4)$ \\
\hline Fe1 & $0.2805(3)$ & 0.25000 & $0.9792(14)$ & $0.990(4)$ \\
\hline P1 & $0.0964(9)$ & 0.25000 & $0.420(2)$ & 1 \\
\hline O1 & $0.098(2)$ & 0.25000 & $0.712(4)$ & 1 \\
\hline O2 & $0.448(3)$ & 0.25000 & $0.220(2)$ & 1 \\
\hline O3 & $0.1572(14)$ & $0.045(3)$ & $0.2730(19)$ & 1 \\
\hline Fe2 & 0.00000 & 0.00000 & 0.00000 & $0.9792(14)$ \\
\hline Li2 & $0.2805(3)$ & 0.25000 & $0.010(4)$ \\
\hline
\end{tabular}


Last frame, $\mathrm{LiFePO}_{4}$, precursor $\mathrm{A}, 345^{\circ} \mathrm{C}$

\begin{tabular}{|l|l|}
\hline $\mathrm{R}_{\text {Bragg }}$ & $4.20 \%$ \\
\hline Number of data points & 1096 \\
\hline Number of background points & 24 \\
\hline Total number of refined parameters & 42 \\
\hline $\mathrm{a} / \AA$ & $10.4008(4)$ \\
\hline $\mathrm{b} / \AA$ & $6.0523(2)$ \\
\hline $\mathrm{c} / \AA$ & $4.7324(2)$ \\
\hline $\mathrm{B}_{\text {overall }} / \AA^{2}$ & $1.61(11)$ \\
\hline $\mathrm{U}$ (peak shape) & $0.71(2)$ \\
\hline
\end{tabular}

Atomic positions:

\begin{tabular}{|l|l|l|l|l|}
\hline Species & X-coordinate & y-coordinate & z-coordinate & Occupancy \\
\hline Li1 & 0.00000 & 0.00000 & 0.00000 & $0.996(4)$ \\
\hline Fe1 & $0.2822(3)$ & 0.25000 & $0.9769(11)$ & $0.996(4)$ \\
\hline P1 & $0.0991(8)$ & 0.25000 & $0.416(2$ & 1 \\
\hline O1 & $0.094(2)$ & 0.25000 & $0.726(4)$ & 1 \\
\hline O2 & $0.458(2)$ & 0.25000 & $0.207(2)$ & 1 \\
\hline O3 & $0.1638(13)$ & $0.046(2)$ & $0.2807(17)$ & 1 \\
\hline Fe2 & 0.00000 & 0.00000 & 0.00000 & $0.010(4)$ \\
\hline Li2 & $0.2822(3)$ & 0.25000 & $0.9769(11)$ & $0.010(4)$ \\
\hline
\end{tabular}

Last frame, $\mathrm{LiFePO}_{4}$, precursor $\mathrm{A}, 3^{\circ}{ }^{\circ} \mathrm{C}$

\begin{tabular}{|l|l|}
\hline $\mathrm{R}_{\text {Bragg }}$ & $2.24 \%$ \\
\hline Number of data points & 1102 \\
\hline Number of background points & 24 \\
\hline Total number of refined parameters & 42 \\
\hline $\mathrm{a} / \AA$ & $10.4008(4)$ \\
\hline $\mathrm{b} / \AA$ & $6.0523(2)$ \\
\hline $\mathrm{c} / \AA$ & $4.7324(2)$ \\
\hline $\mathrm{B}_{\text {overall }} / \AA^{2}$ & $1.61(11)$ \\
\hline $\mathrm{U}$ (peak shape) & $0.71(2)$ \\
\hline
\end{tabular}

\section{Atomic positions:}

\begin{tabular}{|l|l|l|l|l|}
\hline Species & x-coordinate & y-coordinate & z-coordinate & Occupancy \\
\hline Li1 & 0.00000 & 0.00000 & 0.00000 & $0.990(2)$ \\
\hline Fe1 & $0.2835(2)$ & 0.25000 & $0.9742(8)$ & $0.990(2)$ \\
\hline P1 & $0.0970(6)$ & 0.25000 & $0.4228(15)$ & 1 \\
\hline O1 & $0.0964(15)$ & 0.25000 & $0.740(2)$ & 1 \\
\hline O2 & $0.4603(15)$ & 0.25000 & $0.1972(17)$ & 1 \\
\hline O3 & $0.1678(10)$ & $0.0508(15)$ & $0.2824(13)$ & 1 \\
\hline Fe2 & 0.00000 & 0.00000 & 0.00000 & $0.010(2)$ \\
\hline Li2 & $0.2835(2)$ & 0.25000 & $0.9742(8)$ & $0.010(2)$ \\
\hline
\end{tabular}


Last frame, $\mathrm{LiFePO}_{4}$, precursor $\mathrm{B}, \mathrm{170}^{\circ} \mathrm{C}$

\begin{tabular}{|l|l|}
\hline $\mathrm{R}_{\text {Bragg }}$ & $1.60 \%$ \\
\hline Number of data points & 1027 \\
\hline Number of background points & 28 \\
\hline Total number of refined parameters & 47 \\
\hline $\mathrm{a} / \AA$ & $10.3469(11)$ \\
\hline $\mathrm{b} / \AA$ & $6.0062(7)$ \\
\hline $\mathrm{c} / \AA$ & $4.7051(6)$ \\
\hline $\mathrm{B}_{\text {overall }} / \AA^{2}$ & $0.9(3)$ \\
\hline $\mathrm{U}$ (peak shape) & $0.36(9)$ \\
\hline $\mathrm{Y}$ (peak shape) & $0.052(5)$ \\
\hline
\end{tabular}

\section{Atomic positions:}

\begin{tabular}{|l|l|l|l|l|}
\hline Species & x-coordinate & y-coordinate & z-coordinate \\
\hline Li1 & 0.00000 & 0.00000 & 0.00000 \\
\hline Fe1 & $0.2823(8)$ & 0.25000 & $0.975(3)$ \\
\hline P1 & $0.097(2)$ & 0.25000 & $0.419(4)$ & $0.993(12)$ \\
\hline O1 & $0.093(5)$ & 0.25000 & $0.743(7)$ & $0.993(12)$ \\
\hline O2 & $0.450(5)$ & 0.25000 & $0.206(6)$ & 1 \\
\hline O3 & $0.164(4)$ & $0.053(5)$ & $0.283(5)$ & 1 \\
\hline Fe2 & 0.00000 & 0.00000 & 0.00000 & 1 \\
\hline Li2 & $0.2823(8)$ & 0.25000 & $0.975(3)$ \\
\hline
\end{tabular}

\section{Last frame, $\mathrm{LiFePO}_{4}$, precursor $\mathrm{B}, \mathrm{270}^{\circ} \mathrm{C}$}

\begin{tabular}{|l|l|}
\hline $\mathrm{R}_{\text {Bragg }}$ & 1.92 \\
\hline Number of data points & 1092 \\
\hline Number of background points & 28 \\
\hline Total number of refined parameters & 47 \\
\hline $\mathrm{a} / \AA$ & $10.3233(2)$ \\
\hline $\mathrm{b} / \AA$ & $6.0010(1)$ \\
\hline $\mathrm{c} / \AA$ & $4.6970(1)$ \\
\hline $\mathrm{B}_{\text {overall }} / \AA^{2}$ & $1.3(6)$ \\
\hline $\mathrm{U}$ (peak shape) & $0.30(14)$ \\
\hline $\mathrm{Y}$ (peak shape) & $0.03(2)$ \\
\hline
\end{tabular}

Atomic positions:

\begin{tabular}{|l|l|l|l|l|}
\hline Species & x-coordinate & y-coordinate & z-coordinate & Occupancy \\
\hline Li1 & 0.00000 & 0.00000 & 0.00000 & $0.996(23)$ \\
\hline Fe1 & $0.2827(14)$ & 0.25000 & $0.975(6)$ & $0.996(23)$ \\
\hline P1 & $0.096(4)$ & 0.25000 & $0.417(7)$ & 1 \\
\hline O1 & $0.096(10)$ & 0.25000 & $0.738(14)$ & 1 \\
\hline O2 & $0.451(12)$ & 0.25000 & $0.205(12)$ & 1 \\
\hline O3 & $0.165(8)$ & $0.049(12)$ & $0.284(9)$ & 1 \\
\hline Fe2 & 0.00000 & 0.00000 & 0.00000 & $0.040(23)$ \\
\hline Li2 & $0.2827(14)$ & 0.25000 & $0.975(6)$ & $0.040(23)$ \\
\hline
\end{tabular}


Last frame, $\mathrm{LiFePO}_{4}$, precursor $\mathrm{B}, 345^{\circ} \mathrm{C}$

\begin{tabular}{|l|l|}
\hline $\mathrm{R}_{\text {Bragg }}$ & $3.04 \%$ \\
\hline Number of data points & 1092 \\
\hline Number of background points & 28 \\
\hline Total number of refined parameters & 47 \\
\hline $\mathrm{a} / \AA$ & $10.3739(22$ \\
\hline $\mathrm{b} / \AA$ & $6.0312(13)$ \\
\hline $\mathrm{c} / \AA$ & $4.7220(11)$ \\
\hline $\mathrm{B}_{\text {overall }} / \AA^{2}$ & $0.83(58)$ \\
\hline $\mathrm{U}$ (peak shape) & $0.50(10)$ \\
\hline
\end{tabular}

Atomic positions:

\begin{tabular}{|l|l|l|l|l|}
\hline Species & x-coordinate & y-coordinate & z-coordinate & Occupancy \\
\hline Li1 & 0.00000 & 0.00000 & 0.00000 & $0.993(26)$ \\
\hline Fe1 & $0.2823(17)$ & 0.25000 & $0.974(6)$ & $0.993(26)$ \\
\hline P1 & $0.099(4)$ & 0.25000 & $0.421(9)$ & 1 \\
\hline O1 & $0.090(10)$ & 0.25000 & $0.738(17)$ & 1 \\
\hline O2 & $0.452(11)$ & 0.25000 & $0.200(14)$ & 1 \\
\hline O3 & $0.165(7)$ & $0.058(10)$ & $0.281(11)$ & 1 \\
\hline Fe2 & 0.00000 & 0.00000 & 0.00000 & $0.007(26)$ \\
\hline Li2 & $0.2823(17)$ & $0.974(6)$ & $0.007(26)$ \\
\hline
\end{tabular}

\section{Last frame, $\mathrm{LiFe}_{0.75} \mathrm{Mn}_{0.25} \mathrm{PO}_{4},{ }_{170}{ }^{\circ} \mathrm{C}$}

\begin{tabular}{|l|l|}
\hline $\mathrm{R}_{\text {Bragg }}$ & $3.40 \%$ \\
\hline Number of data points & 1086 \\
\hline Number of background points & 25 \\
\hline Total number of refined parameters & 43 \\
\hline $\mathrm{a} / \AA$ & $10.3430(3)$ \\
\hline $\mathrm{b} / \AA$ & $6.0099(2)$ \\
\hline $\mathrm{c} / \AA$ & $4.7039(1)$ \\
\hline $\mathrm{B}_{\text {overall }} / \AA^{2}$ & $0.88(8)$ \\
\hline $\mathrm{U}$ (peak shape) & $0.41(1)$ \\
\hline
\end{tabular}

\section{Atomic positions:}

\begin{tabular}{|l|l|l|l|l|}
\hline Species & x-coordinate & y-coordinate & z-coordinate & Occupancy \\
\hline Li1 & 0.00000 & 0.00000 & 0.00000 & $0.973(2)$ \\
\hline Fe1/Mn1 & $0.2819(2)$ & 0.25000 & $0.9759(8)$ & $0.973(2)$ \\
\hline P1 & $0.0941(6)$ & 0.25000 & $0.4150(11)$ & 1 \\
\hline O1 & $0.1042(13)$ & 0.25000 & $0.7403(19)$ & 1 \\
\hline O2 & $0.4440(17)$ & 0.25000 & $0.2102(15)$ & 1 \\
\hline O3 & $0.1586(9)$ & $0.0430(16)$ & $0.2786(13)$ & 1 \\
\hline Fe2/Mn2 & 0.00000 & 0.00000 & 0.00000 & $0.027(2)$ \\
\hline Li2 & $0.2819(2)$ & 0.25000 & $0.9759(8)$ & $0.027(2)$ \\
\hline
\end{tabular}


Last frame, $\mathrm{LiFe}_{0.75} \mathrm{Mn}_{0.25} \mathrm{PO}_{4} 270$

\begin{tabular}{|l|l|}
\hline $\mathrm{R}_{\text {Bragg }}$ & $1.21 \%$ \\
\hline Number of data points & 1086 \\
\hline Number of background points & 25 \\
\hline Total number of refined parameters & 43 \\
\hline $\mathrm{a} / \AA$ & $10.3706(16)$ \\
\hline $\mathrm{b} / \AA$ & $6.0327(10)$ \\
\hline $\mathrm{c} / \AA$ & $4.7157(8)$ \\
\hline $\mathrm{B}_{\text {overall }} / \AA^{2}$ & $1.0(4)$ \\
\hline $\mathrm{U}$ (peak shape) & $0.3(1)$ \\
\hline $\mathrm{Y}$ (peak shape) & $0.05(2)$ \\
\hline
\end{tabular}

\section{Atomic positions:}

\begin{tabular}{|l|l|l|l|l|}
\hline Species & x-coordinate & y-coordinate & z-coordinate & Occupancy \\
\hline Li1 & 0.00000 & 0.00000 & 0.00000 & $0.997(2)$ \\
\hline Fe1/Mn1 & $0.2826(12)$ & 0.25000 & $0.973(4)$ & $0.997(2)$ \\
\hline P & $0.097(3)$ & 0.25000 & $0.419(6)$ & 1 \\
\hline O1 & $0.095(7)$ & 0.25000 & $0.737(10)$ & 1 \\
\hline O2 & $0.455(8)$ & 0.25000 & $0.204(9)$ & 1 \\
\hline 03 & $0.164(6)$ & $0.050(8)$ & $0.282(7)$ & 1 \\
\hline Fe2/Mn2 & 0.00000 & 0.00000 & 0.00000 & $0.003(2)$ \\
\hline Li2 & $0.2826(12)$ & 0.25000 & $0.973(4)$ & $0.003(2)$ \\
\hline
\end{tabular}

\section{Last frame, $\mathrm{LiFe}_{0.75} \mathrm{Mn}_{0.25} \mathrm{PO}_{4} 345$}

\begin{tabular}{|l|l|}
\hline $\mathrm{R}_{\text {Bragg }}$ & $1.44 \%$ \\
\hline Number of data points & 1086 \\
\hline Number of background points & 25 \\
\hline Total number of refined parameters & 43 \\
\hline $\mathrm{a} / \AA$ & $10.3789(1)$ \\
\hline $\mathrm{b} / \AA$ & $6.0394(1)$ \\
\hline $\mathrm{c} / \AA$ & $4.7210(1)$ \\
\hline $\mathrm{B}_{\text {overall }} / \AA^{2}$ & $1.09(4)$ \\
\hline $\mathrm{U}$ (peak shape) & $0.30(1)$ \\
\hline $\mathrm{Y}$ (peak shape) & $0.043(2)$ \\
\hline
\end{tabular}

\section{Atomic positions:}

\begin{tabular}{|l|l|l|l|l|}
\hline Species & x-coordinate & y-coordinate & z-coordinate & Occupancy \\
\hline Li1 & 0.00000 & 0.00000 & 0.00000 & $0.996(2)$ \\
\hline Fe1/Mn1 & $0.28314(11)$ & 0.25000 & $0.9735(4)$ & $0.996(2)$ \\
\hline P1 & 0.0000 & $0.0968(3)$ & 0.25000 & 1 \\
\hline O1 & $0.0947(6)$ & 0.25000 & $0.7343(10)$ & 1 \\
\hline O2 & $0.4551(7)$ & 0.25000 & $0.2079(9)$ & 1 \\
\hline O3 & $0.1660(5)$ & $0.0496(8)$ & $0.2829(6)$ & 1 \\
\hline Fe2/Mn2 & 0.00000 & 0.00000 & 0.00000 & $0.004(2)$ \\
\hline Li2 & $0.28314(11)$ & 0.25000 & $0.9735(4)$ & $0.004(2)$ \\
\hline
\end{tabular}


Last frame, $\mathrm{LiFe}_{0.75} \mathrm{Mn}_{0.25} \mathrm{PO}_{4} 380$

\begin{tabular}{|l|l|}
\hline $\mathrm{R}_{\text {Bragg }}$ & $1.44 \%$ \\
\hline Number of data points & 1077 \\
\hline Number of background points & 25 \\
\hline Total number of refined parameters & 43 \\
\hline a / $\AA$ & $10.4049(2)$ \\
\hline $\mathrm{b} / \AA$ & $6.0553(1)$ \\
\hline $\mathrm{c} / \AA$ & $4.7351(1)$ \\
\hline $\mathrm{B}_{\text {overall }} / \AA^{2}$ & $0.98(5)$ \\
\hline $\mathrm{U}$ (peak shape) & $0.415(7)$ \\
\hline
\end{tabular}

Atomic positions:

\begin{tabular}{|l|l|l|l|l|}
\hline Species & x-coordinate & y-coordinate & z-coordinate & Occupancy \\
\hline Li1 & 0.00000 & 0.00000 & 0.00000 & $0.992(1)$ \\
\hline Fe1/Mn1 & $0.28283(14)$ & 0.25000 & $0.9735(5)$ & $0.992(1)$ \\
\hline P & $0.0968(3)$ & 0.25000 & $0.4148(7)$ & 1 \\
\hline O1 & $0.0972(8)$ & 0.25000 & $0.7305(13)$ & 1 \\
\hline O2 & $0.4571(9)$ & 0.25000 & $0.2011(11)$ & 1 \\
\hline O3 & $0.1641(7)$ & $0.0469(10)$ & $0.2789(8)$ & 1 \\
\hline Fe2/Mn2 & 0.00000 & 0.00000 & 0.00000 & $0.007(1)$ \\
\hline Li2 & $0.28283(14)$ & 0.25000 & $0.9735(5)$ & $0.007(1)$ \\
\hline
\end{tabular}

\section{Last frame, $\mathrm{LiFe}_{0.50} \mathrm{Mn}_{0.50} \mathrm{PO}_{4} 170$}

\begin{tabular}{|l|l|}
\hline $\mathrm{R}_{\text {Bragg }}$ & $1.05 \%$ \\
\hline Number of data points & 1077 \\
\hline Number of background points & 25 \\
\hline Total number of refined parameters & 43 \\
\hline $\mathrm{a} / \AA$ & $10.4194(2)$ \\
\hline $\mathrm{b} / \AA$ & $6.05381(1)$ \\
\hline $\mathrm{c} / \AA$ & $4.74016(1)$ \\
\hline $\mathrm{B}_{\text {overall }} / \AA^{2}$ & $1.38(5)$ \\
\hline $\mathrm{U}$ (peak shape) & $0.54(1)$ \\
\hline $\mathrm{Y}$ (peak shape) & $0.047(2)$ \\
\hline
\end{tabular}

Atomic positions:

\begin{tabular}{|l|l|l|l|l|}
\hline Species & x-coordinate & y-coordinate & z-coordinate & Occupancy \\
\hline Li1 & 0.00000 & 0.00000 & 0.00000 & $0.973(1)$ \\
\hline Fe1 & $0.28199(11)$ & 0.25000 & $0.9729(4)$ & $0.973(1)$ \\
\hline P1 & $0.0956(3)$ & 0.25000 & $0.4113(6)$ & 1 \\
\hline O1 & $0.0965(7)$ & 0.25000 & $0.7364(12)$ & 1 \\
\hline O2 & $0.4487(9)$ & 0.25000 & $0.2113(8)$ & 1 \\
\hline O3 & $0.1631(5)$ & $0.0439(8)$ & $0.2773(7)$ & 1 \\
\hline Fe2/Mn2 & 0.00000 & 0.00000 & 0.00000 & $0.027(1)$ \\
\hline Li2 & $0.28199(11)$ & 0.25000 & $0.9729(4)$ & $0.027(1)$ \\
\hline
\end{tabular}


Last frame, $\mathrm{LiFe}_{0.50} \mathrm{Mn}_{0.50} \mathrm{PO}_{4} 270^{\circ} \mathrm{C}$

\begin{tabular}{|l|l|}
\hline $\mathrm{R}_{\text {Bragg }}$ & $1.64 \%$ \\
\hline Number of data points & 1077 \\
\hline Number of background points & 25 \\
\hline Total number of refined parameters & 43 \\
\hline $\mathrm{a} / \AA$ & $10.4097(2)$ \\
\hline $\mathrm{b} / \AA$ & $6.0609(1)$ \\
\hline $\mathrm{c} / \AA$ & $4.7332(1)$ \\
\hline $\mathrm{B}_{\text {overall }} / \AA^{2}$ & $1.27(5)$ \\
\hline $\mathrm{U}$ (peak shape) & $0.39(1)$ \\
\hline $\mathrm{Y}$ (peak shape) & $0.155(2)$ \\
\hline
\end{tabular}

Atomic positions:

\begin{tabular}{|l|l|l|l|l|}
\hline Species & x-coordinate & y-coordinate & z-coordinate & Occupancy \\
\hline Li1 & 0.00000 & 0.00000 & 0.00000 & $0.991(1)$ \\
\hline Fe1/Mn1 & $0.28264(13)$ & 0.25000 & $0.9737(5)$ & $0.991(1)$ \\
\hline P1 & $0.0944(4)$ & 0.25000 & $0.4155(7)$ & 1 \\
\hline O1 & $0.0984(8)$ & 0.25000 & $0.7358(13)$ & 1 \\
\hline O2 & $0.4530(10)$ & 0.25000 & $0.2046(9)$ & 1 \\
\hline O3 & $0.1638(6)$ & $0.0492(9)$ & $0.2822(8)$ & 1 \\
\hline Fe2 & 0.00000 & 0.00000 & 0.00000 & $0.009(1)$ \\
\hline Li2 & $0.28264(13)$ & 0.25000 & $0.9737(5)$ & $0.009(1)$ \\
\hline
\end{tabular}

Last frame, $\mathrm{LiFe}_{0.50} \mathrm{Mn}_{0.50} \mathrm{PO}_{4} 345^{\circ} \mathrm{C}$

\begin{tabular}{|l|l|}
\hline $\mathrm{R}_{\text {Bragg }}$ & $3.95 \%$ \\
\hline Number of data points & 1077 \\
\hline Number of background points & 25 \\
\hline Total number of refined parameters & 43 \\
\hline $\mathrm{a} / \AA$ & $10.4072(3)$ \\
\hline $\mathrm{b} / \AA$ & $6.0619(1)$ \\
\hline $\mathrm{c} / \AA$ & $4.7336(1)$ \\
\hline $\mathrm{B}_{\text {overall }} / \AA^{2}$ & $0.94(9)$ \\
\hline $\mathrm{U}$ (peak shape) & $0.48(2)$ \\
\hline $\mathrm{Y}$ (peak shape) & $0.019(2)$ \\
\hline
\end{tabular}

Atomic positions:

\begin{tabular}{|l|l|l|l|l|}
\hline Species & x-coordinate & y-coordinate & z-coordinate & Occupancy \\
\hline Li1 & 0.00000 & 0.00000 & 0.00000 & $0.996(2)$ \\
\hline Fe1/Mn1 & $0.2828(2)$ & 0.25000 & $0.9705(7)$ & $0.996(2)$ \\
\hline P1 & $0.0952(6)$ & 0.25000 & $0.4169(11)$ & 1 \\
\hline O1 & $0.0939(13)$ & 0.25000 & $0.731(2)$ & 1 \\
\hline O2 & $0.4495(15)$ & 0.25000 & $0.2062(14)$ & 1 \\
\hline O3 & $0.1604(8)$ & $0.0537(14)$ & $0.2778(12)$ & 1 \\
\hline Fe2/Mn2 & 0.00000 & 0.00000 & 0.00000 & $0.004(2)$ \\
\hline Li2 & $0.2828(2)$ & 0.25000 & $0.9705(7)$ & $0.004(2)$ \\
\hline
\end{tabular}




\section{Last frame, $\mathrm{LiFe}_{0.25} \mathrm{Mn}_{0.75} \mathrm{PO}_{4} \mathrm{170}^{\circ} \mathrm{C}$}

\begin{tabular}{|l|l|}
\hline $\mathrm{R}_{\text {Bragg }}$ & $3.39 \%$ \\
\hline Number of data points & 1077 \\
\hline Number of background points & 25 \\
\hline Total number of refined parameters & 43 \\
\hline $\mathrm{a} / \AA$ & $10.4125(3)$ \\
\hline $\mathrm{b} / \AA$ & $6.0547(2)$ \\
\hline $\mathrm{c} / \AA$ & $4.7419(2)$ \\
\hline $\mathrm{B}_{\text {overall }} / \AA^{2}$ & $1.36(9)$ \\
\hline $\mathrm{U}$ (peak shape) & $0.62(3)$ \\
\hline $\mathrm{Y}$ (peak shape) & $0.051(3)$ \\
\hline
\end{tabular}

\section{Atomic positions:}

\begin{tabular}{|l|l|l|l|l|}
\hline Species & x-coordinate & y-coordinate & z-coordinate & Occupancy \\
\hline Li1 & 0.00000 & 0.00000 & 0.00000 & $0.967(2)$ \\
\hline Fe1/Mn1 & $0.2822(2)$ & 0.25000 & $0.9704(8)$ & $0.967(2)$ \\
\hline P1 & $0.0932(7)$ & 0.25000 & $0.4091(13)$ & 1 \\
\hline O1 & $0.1009(14)$ & 0.25000 & $0.728(2)$ & 1 \\
\hline O2 & $0.4423(16)$ & 0.25000 & $0.2135(15)$ & 1 \\
\hline O3 & $0.1552(9)$ & $0.0450(16)$ & $0.2733(14)$ & 1 \\
\hline Fe2/Mn2 & 0.00000 & 0.00000 & 0.00000 & $0.032(2)$ \\
\hline Li2 & $0.2822(2)$ & 0.25000 & $0.9704(8)$ & $0.032(2)$ \\
\hline
\end{tabular}

\section{Last frame, $\mathrm{LiFe}_{0.75} \mathrm{Mn}_{0.75} \mathrm{PO}_{4} 270$}

\begin{tabular}{|l|l|}
\hline $\mathrm{R}_{\text {Bragg }}$ & $2.13 \%$ \\
\hline Number of data points & 1077 \\
\hline Number of background points & 25 \\
\hline Total number of refined parameters & 43 \\
\hline $\mathrm{a} / \AA$ & $10.4258(3)$ \\
\hline $\mathrm{b} / \AA$ & $6.0743(1)$ \\
\hline $\mathrm{c} / \AA$ & $4.7448(3)$ \\
\hline $\mathrm{B}_{\text {overall }} / \AA^{2}$ & $1.25(6)$ \\
\hline $\mathrm{U}$ (peak shape) & $0.50(2)$ \\
\hline $\mathrm{Y}$ (peak shape) & $0.041(2)$ \\
\hline
\end{tabular}

\section{Atomic positions}

\begin{tabular}{|l|l|l|l|l|}
\hline Li1 & 0.00000 & 0.00000 & 0.00000 & $0.988(2)$ \\
\hline Fe/Mn1 & $0.28236(15)$ & 0.25000 & $0.9707(6)$ & $0.988(2)$ \\
\hline P1 & $0.0941(4)$ & 0.25000 & $0.4122(9)$ & 1 \\
\hline O1 & $0.0987(9)$ & 0.25000 & $0.7291(16)$ & 1 \\
\hline O2 & $0.4469(11)$ & 0.25000 & $0.2076(11)$ & 1 \\
\hline O3 & $0.1581(6)$ & $0.0499(11)$ & $0.2755(9)$ & 1 \\
\hline Fe2/Mn2 & 0.00000 & 0.00000 & 0.00000 & $0.012(2)$ \\
\hline Li2 & $0.28236(15)$ & 0.25000 & $0.9707(6)$ & $0.012(2)$ \\
\hline
\end{tabular}


Last frame, $\mathrm{LiFe}_{0.75} \mathrm{Mn}_{0.75} \mathrm{PO}_{4} 345^{\circ} \mathrm{C}$

\begin{tabular}{|l|l|}
\hline $\mathrm{R}_{\text {Bragg }}$ & Abstract $3.57 \%$ \\
\hline Number of data points & 1077 \\
\hline Number of background points & 25 \\
\hline Total number of refined parameters & 43 \\
\hline $\mathrm{a} / \AA$ & $10.4303(3)$ \\
\hline $\mathrm{b} / \AA$ & $6.0808(2)$ \\
\hline $\mathrm{c} / \AA$ & $4.7453(2)$ \\
\hline $\mathrm{B}_{\text {overall }} / \AA^{2}$ & $1.0213(7)$ \\
\hline $\mathrm{U}$ (peak shape) & $0.44(2)$ \\
\hline Y (peak shape) & $0.019(2)$ \\
\hline
\end{tabular}

\section{Atomic positions:}

\begin{tabular}{|l|l|l|l|l|}
\hline Li1 & 0.00000 & 0.00000 & 0.00000 & $0.992(2)$ \\
\hline Fe1/Mn1 & $0.28274(17)$ & 0.25000 & $0.9712(6)$ & $0.992(2)$ \\
\hline P1 & $0.0944(5)$ & 0.25000 & $0.4155(10)$ & 1 \\
\hline O1 & $0.0988(11)$ & 0.25000 & $0.7297(18)$ & 1 \\
\hline O2 & $0.4542(12)$ & 0.25000 & $0.2048(12)$ & 1 \\
\hline O3 & $0.1600(7)$ & $0.0515(12)$ & $0.2797(10)$ & 1 \\
\hline Fe2 & 0.00000 & 0.00000 & 0.00000 & $0.008(2)$ \\
\hline Li2 & $0.28274(17)$ & 0.25000 & $0.9712(6)$ & $0.008(2)$ \\
\hline
\end{tabular}


Figure S3: Initial data frames obtained in the $\mathrm{LiFe}_{0.50} \mathrm{Mn}_{0.50} \mathrm{PO}_{4}$ experiment at $T=170^{\circ} \mathrm{C}$. The time between the frames is 9 seconds.

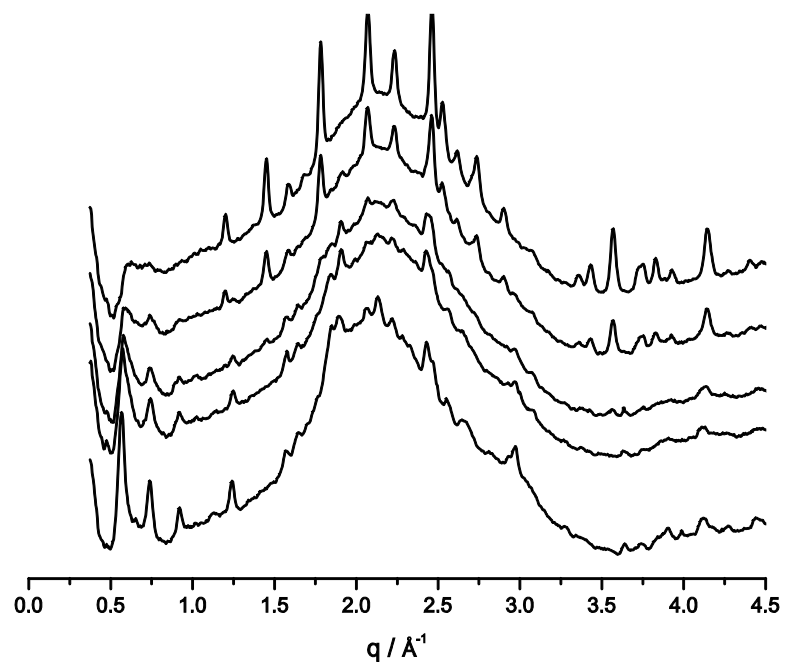

Figure S4: Initial data frames obtained in the $\mathrm{LiFe}_{0.25} \mathrm{Mn}_{0.75} \mathrm{PO}_{4}$ experiment at $T=170^{\circ} \mathrm{C}$. The time between the frames is 9 seconds.

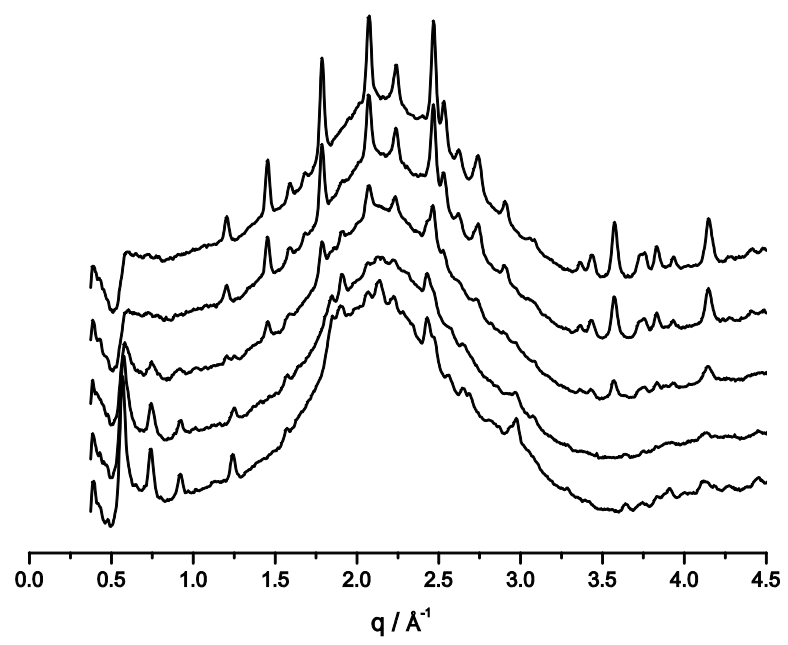


Figure S5. Changes in the unit cell parameters for $\mathrm{LiFe}_{0.75} \mathrm{Mn}_{0.25} \mathrm{PO}_{4}$. For clarity, only every third data point is shown. Squares show the $a$ axis, circles the $b$ axis and triangles the $c$ axis.
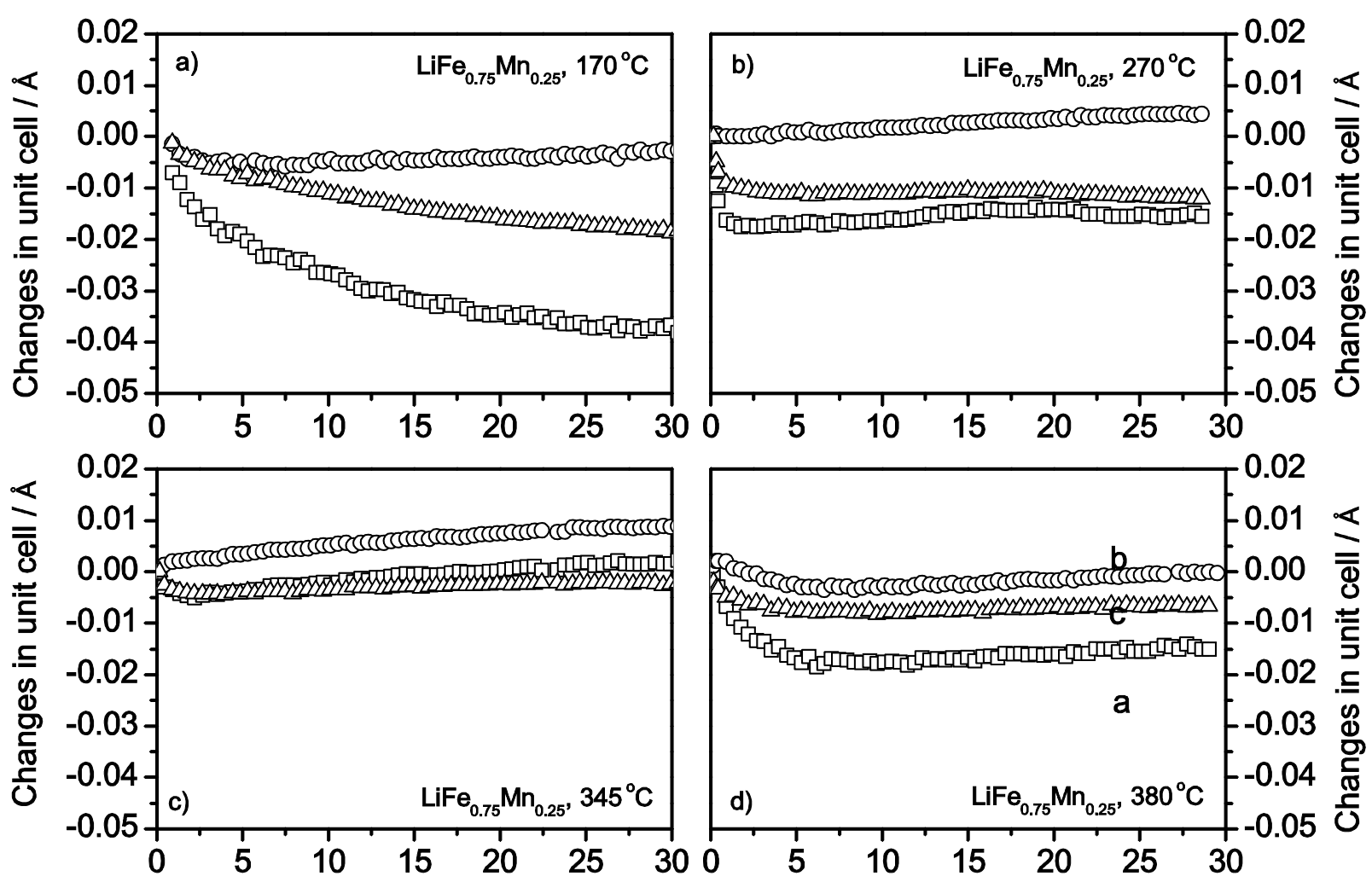
Figure S6. Changes in the unit cell parameters for $\mathrm{LiFe}_{0.50} \mathrm{Mn}_{0.50} \mathrm{PO}_{4}$. For clarity, only every third data point is shown. Squares show the $a$ axis, circles the $b$ axis and triangles the $c$ axis.
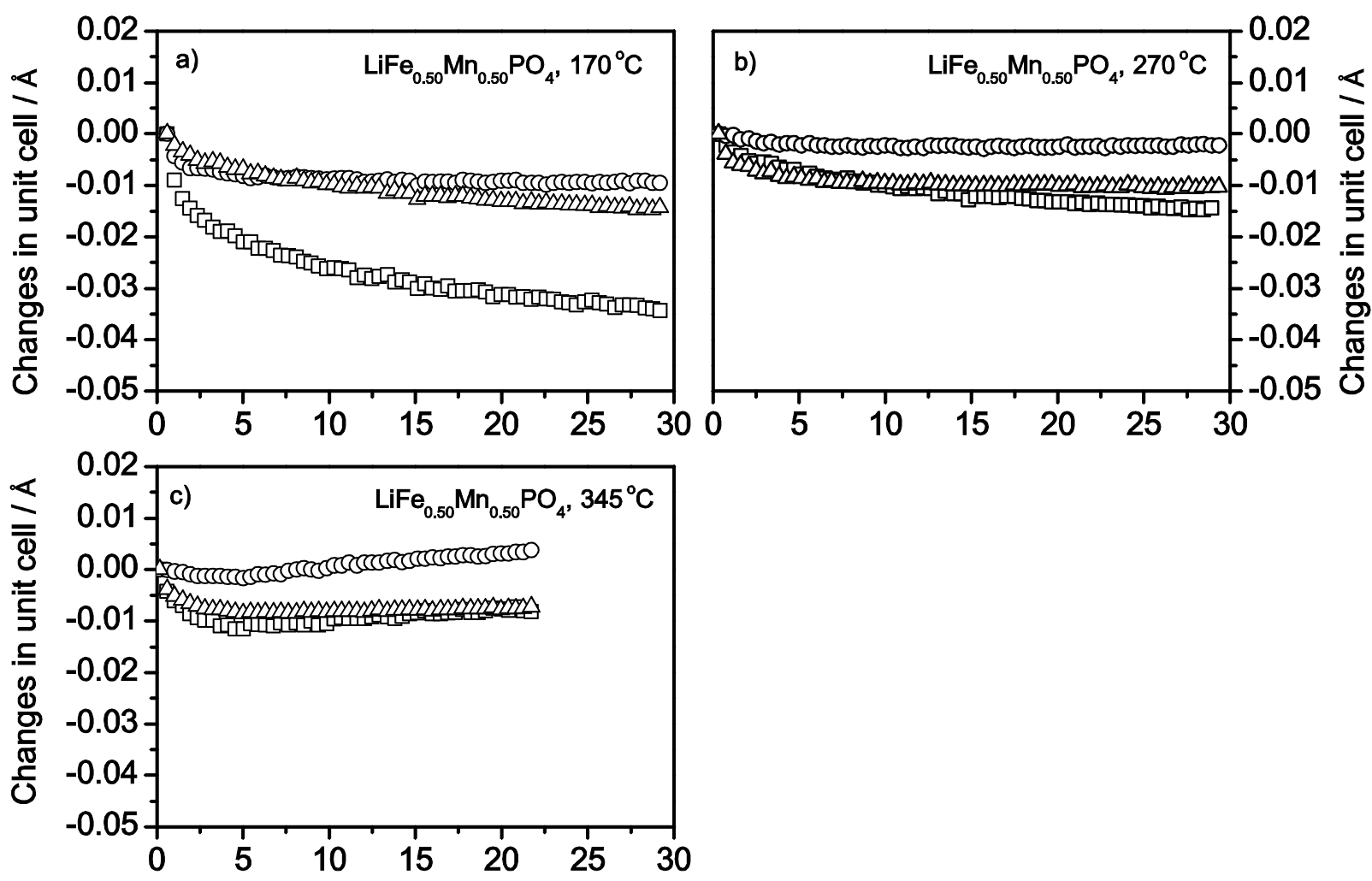
Figure S7: Cation disorder plotted as a function of changes in the unit cell $a$ parameter for $\mathrm{LiFe}_{0.50} \mathrm{Mn}_{0.50} \mathrm{PO}_{4}$. Squares represent data at $T=170{ }^{\circ} \mathrm{C}$, circles at $T=270{ }^{\circ} \mathrm{C}$ and triangles at $T=345{ }^{\circ} \mathrm{C}$

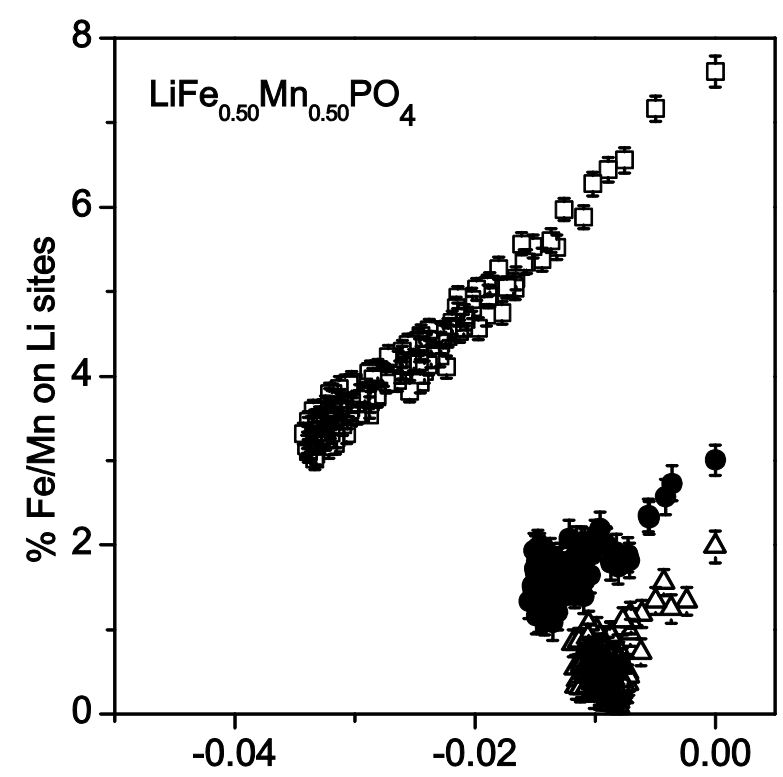

Deviation from initial $a / \AA$

Figure S8. Changes in the unit cell parameters for $\mathrm{LiFe}_{0.25} \mathrm{Mn}_{0.75} \mathrm{PO}_{4}$. For clarity, only every third data point is shown. Squares show the $a$ axis, circles the $b$ axis and triangles the $c$ axis.
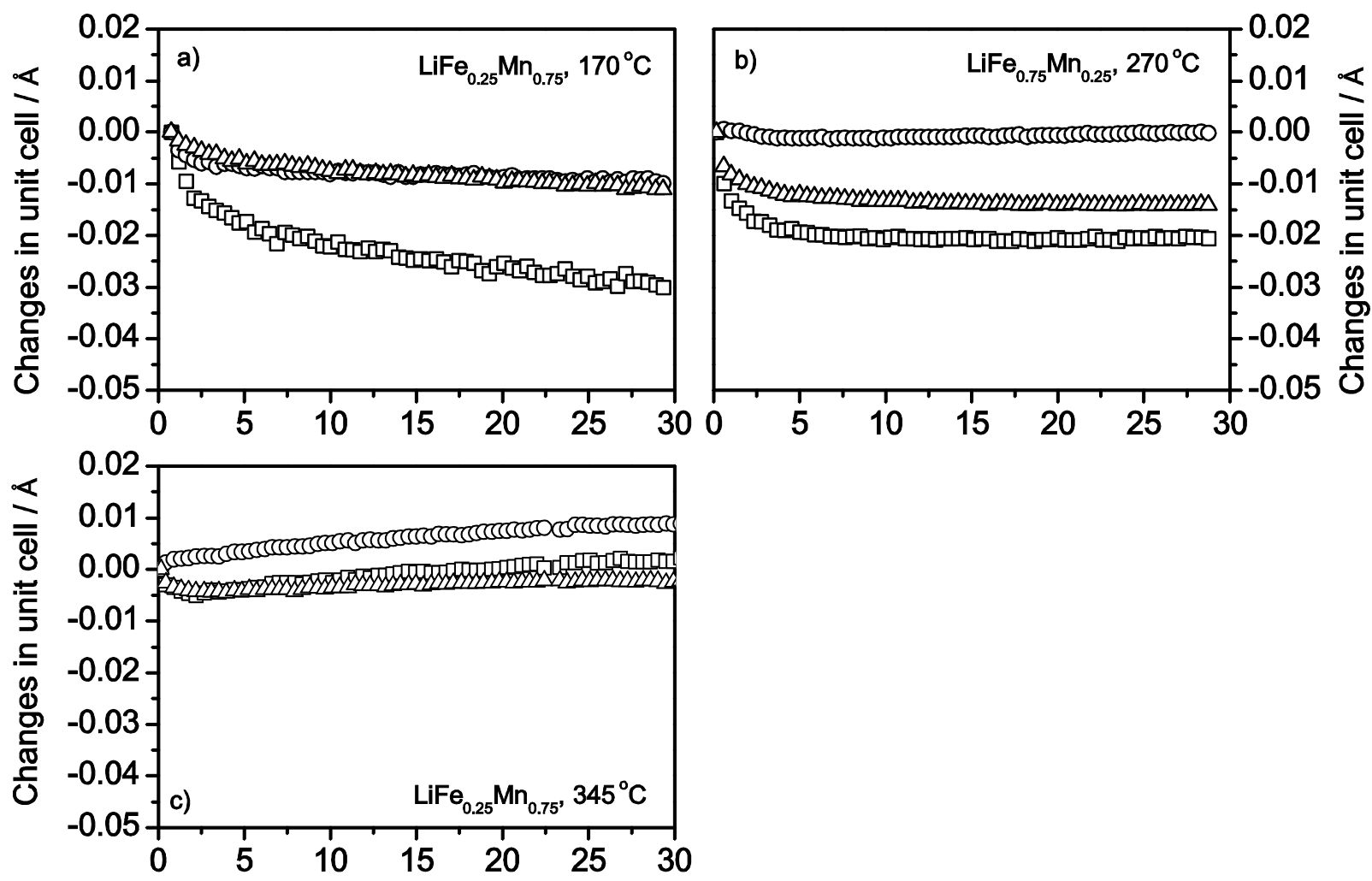
Figure S9: Cation disorder plotted as a function of changes in the unit cell $a$ parameter for

$\mathrm{LiFe}_{0.50} \mathrm{Mn}_{0.50} \mathrm{PO}_{4}$. Squares represent data at $T=170{ }^{\circ} \mathrm{C}$, circles at $T=270{ }^{\circ} \mathrm{C}$ and triangles at $T=345{ }^{\circ} \mathrm{C}$

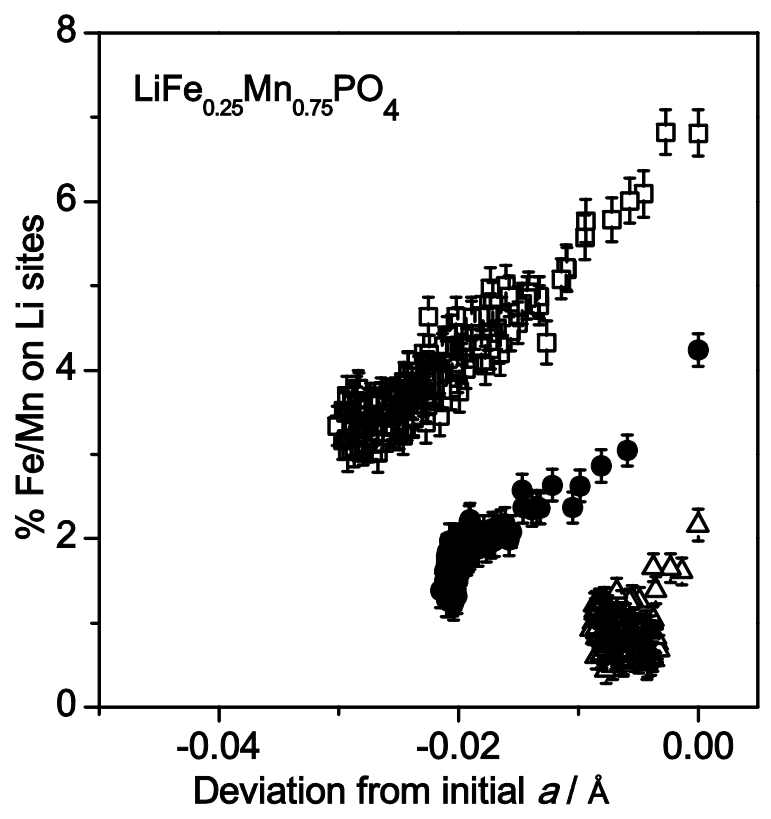

